\title{
Measurement of the Air Fluorescence Yield with the AirLight Experiment
}

\author{
T. Waldenmaier ${ }^{a}$, J. Blümer ${ }^{a, b}$, B. Keilhauer ${ }^{b}$, H. Klages ${ }^{a}$ and S. Klepser ${ }^{c}$ \\ (a) Forschungszentrum Karlsruhe, Institut für Kernphysik, P.O. Box 3640, 76021 Karlsruhe, Germany \\ (b) Universität Karlsruhe (TH), Institut für Experimentelle Kernphysik, P.O. Box 3640, 76021 Karlsruhe, Germany \\ (c) DESY Zeuthen, Platanenallee 6, 15738 Zeuthen, Germany \\ Presenter: B. Keilhauer (bianca.keilhauer@ik.fzk.de), ger-keilhauer-B-abs3-he15-poster
}

For the detection of ultrahigh energy cosmic rays, many experiments rely on the fluorescence technique to measure the longitudinal development of extensive air showers in the atmosphere. The key quantity for the energy reconstruction of the primary particle is the fluorescence yield which depends on pressure, temperature as well as the composition (e.g. water vapor) of the air. The AirLight Experiment at Forschungszentrum Karlsruhe aims for a measurement of the fluorescence yield using electrons emitted by a ${ }^{90} \mathrm{Sr}$ source. The usable energy of the electrons ranges from $250 \mathrm{keV}$ to $2 \mathrm{MeV}$. Different gas mixtures can be tested at pressures between $5 \mathrm{hPa}$ and $1000 \mathrm{hPa}$. The current status and first results will be presented.

\section{Introduction}

Due to the extremely low particle fluxes, very-high energy cosmic rays can be detected only indirectly by observing extensive air showers (EAS). Apart from their detection by large detector arrays on the ground, very-high energy EAS can also be measured with the aid of their fluorescence light emissions. This technique utilizes the atmosphere as a scintillator with the advantage of being able to directly access fundamental shower parameters, as the longitudinal development of the total electromagnetic energy deposit along the shower axis, without relying on theoretical interaction models. Therefore the longitudinal shower profile provides complementary information to the lateral particle distributions at ground, measured by the large detector fields. Challenging is the need of a very good understanding of the entire fluorescence detector, including the atmosphere and the fluorescence emission process.

If all the fluorescence photons are assumed to be emitted along the shower axis, the number of observed photons in the detector $d N_{\gamma}$ per unit path length $d x$ is obtained by

$$
\frac{d N_{\gamma}}{d x}=\int \frac{d^{2} N_{\gamma}^{0}}{d \lambda d x} \cdot \varepsilon_{\mathrm{atm}}(\lambda, x) \cdot \varepsilon_{\mathrm{FD}}(\lambda, x) d \lambda,
$$

where $\varepsilon_{\text {atm }}$ and $\varepsilon_{\mathrm{FD}}$ are the total efficiencies of the atmosphere and the fluorescence detector respectively, which have to be monitored very carefully. The number of produced fluorescence photons at the shower axis $d N_{\gamma}^{0}$ per wavelength bin $d \lambda$ is assumed to be related to the deposited energy $d E_{d e p}$ in the layer $d x$ according to $[1,2]$ :

$$
\frac{d^{2} N_{\gamma}^{0}}{d \lambda d x}=y(\lambda, T, p) \cdot \frac{d E_{\mathrm{dep}}}{d x}
$$

The quantity $y(\lambda, T, p)$ is the fluorescence yield, which depends on the wavelength $\lambda$ of the emitted fluorescence light as well as on temperature $T(x)$ and pressure $p(x)$ of the air at the position of emission. If Eq. (2) is valid, the fluorescence yield $y(\lambda, T, p)$ does not depend on the energy of the ionizing particles and Eq. (1) transforms to the simple form

$$
\frac{d N_{\gamma}}{d x}=\frac{d E_{\mathrm{dep}}}{d x} \int y(\lambda, T, p) \cdot \varepsilon_{\mathrm{atm}}(\lambda, x) \cdot \varepsilon_{\mathrm{FD}}(\lambda, x) d \lambda .
$$

Thus, the fluorescence light output of an EAS is directly related to the energy deposited along its shower axis. 


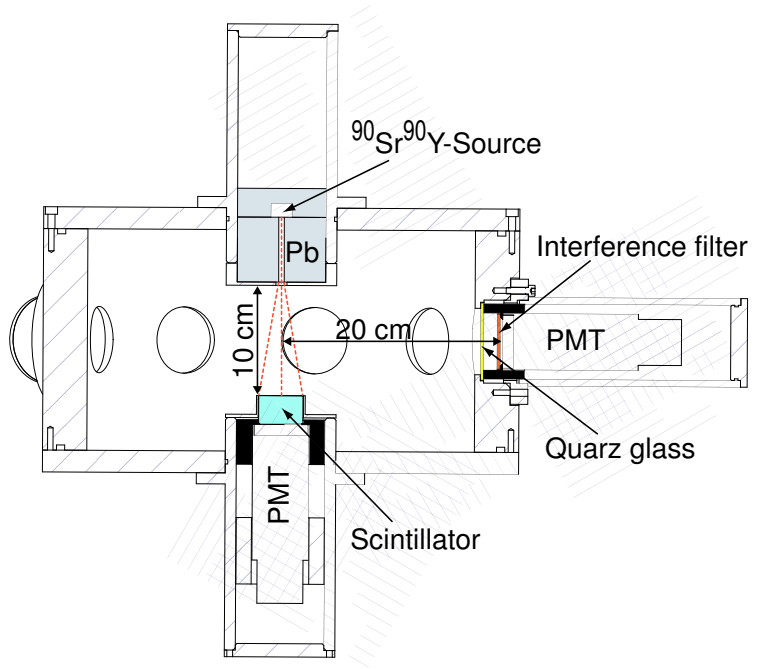

Figure 1. Experimental setup of the AirLight Experiment.

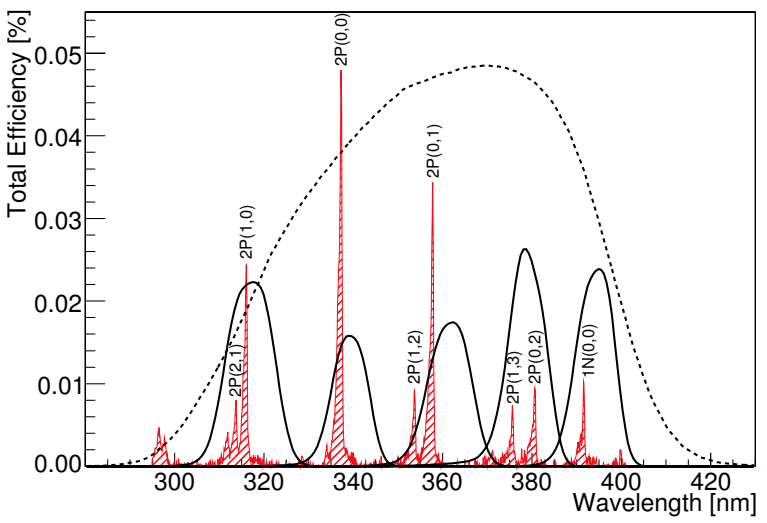

Figure 2. Total efficiencies of the different wavelength bands. The dashed line belongs to the M-UG6 filter and the solid lines represent the interference filters. The most prominent nitrogen transitions are labeled in the sketched spectrum measured by Ulrich et al. [4] (not calibrated).

\section{The AirLight Experiment}

Most of the fluorescence emissions in air, in the wavelength range between $300 \mathrm{~nm}$ and $400 \mathrm{~nm}$, are originating from excited $\mathrm{N}_{2}$ and $\mathrm{N}_{2}^{+}$molecules [3]. In an EAS nearly all of the nitrogen excitations are caused by electrons and positrons with energies below $1 \mathrm{GeV}$ [2]. About $13 \%$ of the deposited energy in the atmosphere comes from low-energy electrons between $250 \mathrm{keV}$ and $2 \mathrm{MeV}$ [2]. The aim of the AirLight Experiment is to measure the relation between the fluorescence emission and the ionization energy deposit of electrons, Eq. (2), in this energy regime for atmospheric pressures, ranging from $5 \mathrm{hPa}$ to $1000 \mathrm{hPa}$. Furthermore, the influence of water vapor will be investigated.

In Fig. 1, the experimental setup of the AirLight experiment is shown. The experiment consists of a cylindrical aluminum chamber with seven photomultipliers (PMT) mounted perpendicular to the chamber axis at a radius of $20 \mathrm{~cm}$. Six PMTs are equipped with narrow band interference filters (FWHM $\sim 10 \mathrm{~nm}$ ) matched to the most important nitrogen transition bands as it is illustrated in Fig. 2. One PMT has a M-UG6 absorption filter as it is used in the fluorescence detectors of the Pierre Auger Observatory [5] to measure the integral fluorescence spectrum between $300 \mathrm{~nm}$ and $420 \mathrm{~nm}$. The chamber is black anodized to suppress photons scattered off the chamber walls which would bias the acceptance of the PMTs. The electrons are emitted from a ${ }^{90} \mathrm{Sr}^{-90} \mathrm{Y}$ beta source with an endpoint energy of $2.3 \mathrm{MeV}$. The source has an activity of $37 \mathrm{MBq}$ and is located behind a lead collimator of $6 \mathrm{~cm}$ length at the top of the chamber. Once the electrons have passed the collimator they traverse $10 \mathrm{~cm}$ of test gas (normally air or pure nitrogen) and are finally stopped in a scintillation detector at the bottom of the chamber. The scintillator measures the energy of the electrons and was calibrated by the measurement of the two well-known Compton-edges in the energy spectrum of a ${ }^{22} \mathrm{Na}$ gamma emitter. Analyzing the smearing of the Compton spectrum, the energy resolution of the scintillator turned out to be about $(E / \mathrm{MeV})^{-0.5} \cdot 10 \%$.

The measuring procedure takes advantage of the coincidence between the electron signal in the scintillator and the signal of the induced fluorescence photons. The resulting distribution of time differences between the electrons and the coincident PMT signals is shown in Fig. 3 for the $337 \mathrm{~nm}$ band. Since the relaxation of the excited states follows an exponential decay law, the signal can be fitted with a Gauss convoluted exponential function taking into account the experimental time resolution of about $0.8 \mathrm{~ns}$. Accidental coincidences can be 


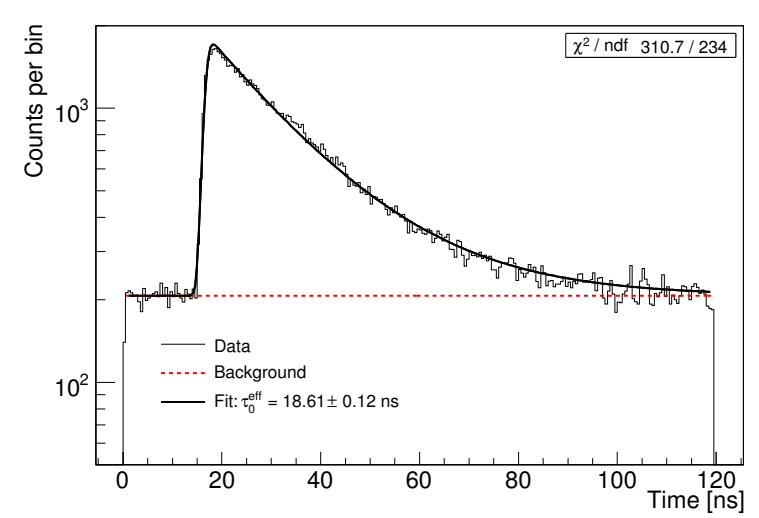

Figure 3. Fluorescence signal of the $2 \mathrm{P}(0,0)$ transition (337 $\mathrm{nm}$ band) in pure nitrogen at a pressure of $100 \mathrm{hPa}$ and $20{ }^{\circ} \mathrm{C}$.

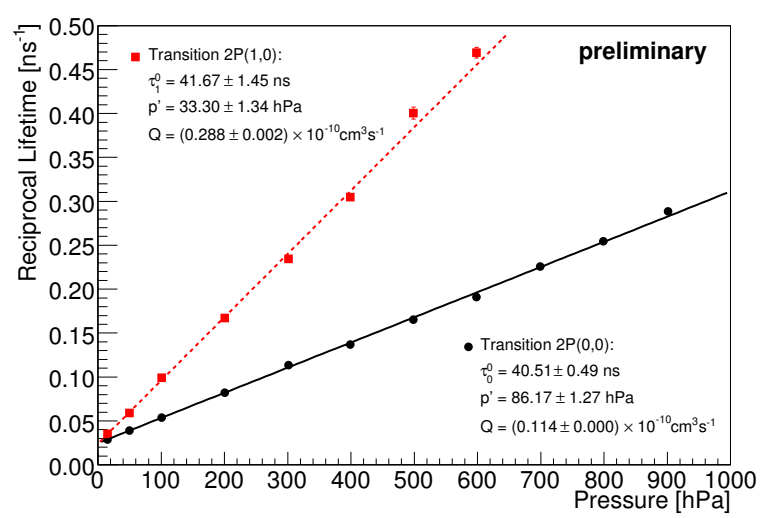

Figure 4. Reciprocal lifetimes $1 / \tau_{v^{\prime}}^{\text {eff }}$ for the $2 \mathrm{P}(0,0)$ and $2 \mathrm{P}(1,0)$ transitions in pure nitrogen vs. pressure at $20{ }^{\circ} \mathrm{C}$. The Q-values are the quenching rate constants for the nitrogen self-quenching.

subtracted as flat background from the fluorescence signals because of their missing time correlation with the scintillator signal.

\section{Some theory and first results}

The data taking and the analysis are still ongoing. Nevertheless, some first results will be discussed in this section. As one can see in Fig. 2, the nitrogen spectrum consists of a variety of bands. Each band belongs to a transition between the vibrational states of a certain electronic transition. For example $2 \mathrm{P}(0,1)$ labels the transition between the vibrational states $v^{\prime}=0$ and $v^{\prime \prime}=1$ of the second positive electronic nitrogen transition system $\left(C^{3} \Pi_{u} \rightarrow B^{3} \Pi_{g}\right)$. The broadening of the bands is due to the rotational substructure which is not resolved in Fig. 2. The relative intensities of the single vibrational bands, which belong to a certain electronic transition, are given by the Einstein transition probabilities $A_{v^{\prime}, v^{\prime \prime}}$ which have been calculated by Gilmore et al. [6]. Therefore the radiative lifetime $\tau_{v^{\prime}}$ of a vibrational state $v^{\prime}$ can be calculated from the sum over all radiative transition probabilities $1 / \tau_{v^{\prime}}=\sum_{v^{\prime \prime}} A_{v^{\prime}, v^{\prime \prime}}$.

Since the excited nitrogen molecules suffer collisions with other molecules, there is a competition between radiative transitions and radiationless deactivations (quenching). The probability of collisional deactivation of the state $v^{\prime}$ can be expressed by $1 / \tau_{v^{\prime}}^{c}$, where $\tau_{v^{\prime}}^{c}$ is the mean time between such collisions. $\tau_{v^{\prime}}^{c}$ depends on the collisional cross sections as well as on temperature $T$ and pressure $p$ of the gas and hence it follows from kinetic gas theory $\tau_{v^{\prime}}^{c} \propto \sqrt{T} / p$ [3]. The relaxation rate $\frac{d N_{v^{\prime}}}{d t}$ and the effective lifetime $\tau_{v^{\prime}}^{\text {eff }}(p, T)$ of an exited state are therefore:

$$
\frac{d N_{v^{\prime}}}{d t}=-\left(\frac{1}{\tau_{v^{\prime}}^{0}}+\frac{1}{\tau_{v^{\prime}}^{c}}\right) \cdot N_{v^{\prime}}(t), \quad \tau_{v^{\prime}}^{\mathrm{eff}}=\frac{\tau_{v^{\prime}}^{0} \tau_{v^{\prime}}^{c}}{\tau_{v^{\prime}}^{0}+\tau_{v^{\prime}}^{c}}=\frac{\tau_{v^{\prime}}^{0}}{1+\frac{p}{p_{v^{\prime}}^{\prime}}} .
$$

$\tau_{v^{\prime}}^{0}$ is the observed lifetime at zero pressure which has not to be necessarily equal to the radiative lifetime $\tau_{v^{\prime}}$, due to internal quenching effects. If the pressure $p$ reaches the reference pressure $p_{v^{\prime}}^{\prime}$, the collisional deactivations are getting stronger than the optical transitions. The probability for an excited state $v^{\prime}$ to relax into a lower state $v^{\prime \prime}$ of the band system through the emission of an optical photon results to be $A_{v^{\prime}, v^{\prime \prime}} \cdot \tau_{v^{\prime}}^{\text {eff }}(p, T)$. 
If the number of excited states is proportional to the local deposited energy $\left(N_{v^{\prime}}=f_{v^{\prime}} \cdot E_{d e p}\right)$, the fluorescence yield of the transition $v^{\prime} \rightarrow v^{\prime \prime}$ can be expressed as

$$
y\left(v^{\prime} \rightarrow v^{\prime \prime}, p, T\right)=f_{v^{\prime}} \cdot A_{v^{\prime}, v^{\prime \prime}} \cdot \tau_{v^{\prime}}^{\text {eff }}(p, T),
$$

where $f_{v^{\prime}}$ is the number of excited states $v^{\prime}$ per deposited energy. As one can see in Fig. 2, the fluorescence spectrum of nitrogen in the wavelength range between $300 \mathrm{~nm}$ and $400 \mathrm{~nm}$ consists almost entirely of vibrational transitions within the second positive system (2P) with $v^{\prime}=0,1$ and the first negative system $(1 \mathrm{~N})$ with $v^{\prime}=0$. Thus the total nitrogen fluorescence yield can be expressed in first approximation as the superposition of three contributions

$$
y_{t o t}(p, T) \approx \sum_{v^{\prime \prime}} y_{2 P}\left(0 \rightarrow v^{\prime \prime}, p, T\right)+y_{2 P}\left(1 \rightarrow v^{\prime \prime}, p, T\right)+y_{1 N}\left(0 \rightarrow v^{\prime \prime}, p, T\right),
$$

where all the transitions of one contribution have the same quenching behavior. Since in Eq. (6) there are only contributions with $v^{\prime}=0,1$ it is sufficient to study the quenching just for the $2 \mathrm{P}(0,0), 2 \mathrm{P}(1,0)$ and $1 \mathrm{~N}(0,0)$ bands. This is shown in Fig. 4, where the reciprocal lifetimes $1 / \tau_{v^{\prime}}^{\text {eff }}$ for the $2 \mathrm{P}$ system are plotted against the

pressure in pure nitrogen. For the $2 \mathrm{P}(1,0)$ transition the contribution of the other two bands in the filter range is not taken into account because of their rather low intensities. The values obtained for this two transitions are in good agreement with other published data [7, 8]. Some filters, especially the filters at $360 \mathrm{~nm}$ and $380 \mathrm{~nm}$, happen to match with two nitrogen bands. For this cases, the fit shown in Fig. 3 has to be done with two components using fixed lifetimes taken from the $2 \mathrm{P}(0,0)$ and $2 \mathrm{P}(1,0)$ data. The measurement of the $1 \mathrm{~N}$ bands is much more complicated because of their large lifetimes at low pressures and their low intensities at high pressures. The corresponding analysis for this transition is still ongoing.

\section{Status and Outlook}

The experimental setup of the AirLight Experiment at Forschungszentrum Karlsruhe has almost been completed. First measurements have been performed in pure nitrogen at $20^{\circ} \mathrm{C}$. The data analysis is in progress and first results about the quenching mechanism are in agreement with previous data. Future work will concentrate on the measurement of the quenching constants for different air constituents and on the absolute calibration of the PMTs, to be able to determine the excitation yields $f_{v^{\prime}}$. In addition GEANT4-simulations [9] of the energy deposition in the chamber and the PMT acceptances are in progress.

Acknowledgements: The authors thank Günter Wörner who constructed the chamber and provided excellent expertise in technical matters.

\section{References}

[1] M. Nagano et al.: Astropart. Phys. 20, 293 (2003)

[2] M. Risse, D. Heck: Astropart. Phys. 20, 661 (2004)

[3] A.N. Bunner: Cosmic Ray Detection by Atmospheric Fluorescence. PhD Thesis, Cornell University, Ithaca, New York (1967)

[4] A. Ulrich: private communication

[5] Auger Collab., J. Abraham et al.: Nucl. Inst. and Meth. A 523, 50 (2004)

[6] F.R. Gilmore, R.R. Laher, P.J. Espy: J. Phys. Chem. Ref. Data 21, 1005 (1992)

[7] S.V. Pancheshnyi et al.: J. Chem. Phys. 262, 349 (2000)

[8] A. Morozov, A. Ulrich et al.: Eur. Phys. J. D 33, 207 (2005)

[9] Geant4 Collab., S. Agostinelli et al.: Nucl. Inst. and Meth. A 506, 250 (2003) 